\title{
MONOPOLAR ELECTROCAUTERY VS SURGICAL CLIPS IN CONTROL OF CYSTIC ARTERY IN LAPAROSCOPIC CHOLECYSTECTOMY: A COMPARATIVE STUDY
}

\author{
Ridipta Sekhar Das ${ }^{1}$, Mrinal Talukdar², Abdul Rahim Shaan ${ }^{3}$ \\ ${ }_{1}^{1}$ Registrar, Department of General Surgery, Silchar Medical College \& Hospital. \\ ${ }^{2}$ Assistant Professor, Department of General Surgery, Silchar Medical College \& Hospital. \\ 3 Post Graduate Resident, Department of General Surgery, Silchar Medical College \& Hospital.
}

\begin{abstract}
BACKGROUND

Laparoscopic cholecystectomy has been extensively accepted since Mouret first successfully introduced the procedure in 1987. During this procedure the cystic artery can be controlled using surgical clips, harmonic scalpel and ligature or monopolar cautery. The extensive use of surgical clips in laparoscopic surgery has led to a variety of complications. Monopolar electrocoagulation can be used to control the cystic artery as it is cheap and universally available. Hence in this study, we compared monopolar electrocautery with clip application for securing haemostasis and to identify the safest and least complicated way for haemostasis of the cystic artery in laparoscopic cholecystectomy.
\end{abstract}

\section{METHODS}

A retrospective analysis of 201 patients were done who were planned for laparoscopic cholecystectomy. Among them 3 were converted to open cholecystectomy due to intraoperative bleeding. The rest 198 patients underwent successful laparoscopic cholecystectomy. In 42 patients the cystic artery was ligated using Ligaclip 300, while in 156 patients the artery was coagulated using monopolar cautery with hook. The patients were observed for any incidences of post-operative haemorrhage and bile leak, difference in length of hospital stay and post-operative complications.

\section{RESULTS}

The mean age was 40.26 years with M:F ratio 1:4. About 86\% (135) and 88\% (37) patients, respectively in electrocautery and Ligaclip group were discharged on the first post-operative day itself. Only 3 (1.5\%) patients, 2 in electrocautery and 1 in Ligaclip group developed post-operative port site infection. These differences were not statistically significant.

\section{CONCLUSION}

We conclude that monopolar electrocautery can be used as a safer alternative to surgical clips in control of cystic artery, especially in developing countries.

\section{KEYWORDS}

Laparoscopic Cholecystectomy, Surgical Clips, Monopolar Electrocautery, Cystic Artery.

HOW TO CITE THIS ARTICLE: Das RS, Talukdar M, Shaan AR. Monopolar electrocautery vs surgical clips in control of cystic artery in laparoscopic cholecystectomy: a comparative study. J. Evolution Med. Dent. Sci. 2016;5(33):1790-1793,

DOI: $10.14260 /$ jemds/2016/422

\section{INTRODUCTION}

In 1985, the first endoscopic cholecystectomy was performed by Mühe of Böblingen, Germany. Although meeting early skepticism from the academic surgical community, Laparoscopic Cholecystectomy (LC) was adopted rapidly around the world and has subsequently been recognized as the new "Gold standard" for the treatment of gallstone disease.[1],[2] In 1992, the National Institutes of Health (NIH) Consensus Development Conference stated that LC "Provides a safe and effective treatment for most patients with symptomatic gallstones.[3]" The advantages of laparoscopic cholecystectomy over open cholecystectomy were immediately appreciated: earlier return of bowel function,

Financial or Other, Competing Interest: None.

Submission 22-03-2016, Peer Review 04-04-2016,

Acceptance 06-04-2016, Published 22-04-2016.

Corresponding Author:

Dr. Abdul Rahim Shaan,

Post Graduate Resident,

Department of General Surgery

Silchar Medical College \& Hospital

Assam.

E-mail:abdulrahimshaan013@gmail.com

DOI: $10.14260 /$ jemds $/ 2016 / 422$ less postoperative pain, improved cosmetics, shorter length of hospital stay, earlier return to full activity, and decreased overall cost.[4] There has been an increase in the rate of cholecystectomies subsequent to the introduction of LC accompanied by evidence of lower clinical thresholds for operative therapy of gallstones.[5] Currently, it is estimated that $90 \%$ of cholecystectomies are performed by the laparoscopic approach. Indeed, LC as a now mature mode of therapy has introduced the general surgical world to the revolutionary advantages and unique perspectives and concerns of minimal access surgery.[6]

Initially, this new technique was associated with a significant increase in morbidity, in particular iatrogenic biliary injuries and arterial haemorrhage. Anatomical relations on laparoscopic visualization are seen differently as compared to open surgery. So the surgeon has to rely on his sound knowledge of the variations in Calot's triangle and do meticulous dissection in order to avoid injury to the extrahepatic biliary tree. ${ }^{[7,8]}$ The course and length of the cystic artery in the Calot's triangle is variable.[9,10] In majority of cases cystic artery arises from right hepatic artery, in few cases 
it may arise from common hepatic, coeliac trunk, right gastric, superior mesenteric, etc. arteries.

During this procedure, proper haemostasis of cystic artery is a must because it may result in torrential haemorrhage if not ligated properly and is the common cause of post-operative bleeding after laparoscopic cholecystectomy. The cystic artery can be controlled using surgical clips, harmonic scalpel and ligature or monopolar cautery. The extensive use of surgical clips in laparoscopic surgery has led to a variety of complications. Clips can slip, dislodge, ulcerate, migrate, internalize and give rise to necrosis of cystic duct with the risk of bile leakage and other complications.[11] Due to the cost issues, harmonic scalpel has been less frequently used for this purpose. Monopolar electrocoagulation can be used to control the cystic artery as it is cheap and universally available. But the use of monopolar electrocautery in the Calot's triangle is still considered taboo by most surgeons. Hence in this study, we compared monopolar electrocautery with clip application for securing haemostasis and to identify the safest and least complicated way for haemostasis of the cystic artery in laparoscopic cholecystectomy.

\section{MATERIALS AND METHODS}

A retrospective analysis of 201 patients were done who were planned for laparoscopic cholecystectomy in the Department of Surgery, Silchar Medical College and Hospital in the time period March 2015 - March 2016.

The approval of Institutional Ethical Committee was obtained.

After admission all patients were subjected to full history and examination and investigations; routine lab tests, routine chest X-ray, ECG, liver function tests, prothrombin time. Abdominal ultrasonography, additional investigations if needed sometimes in some doubtful cases as CT, MRCP and Cholangiogram were done as required.

After pre-operative workups, patients were subjected to surgery.

All patients were operated under elective setting under general anaesthesia. Among them 3 were converted to open cholecystectomy due to intraoperative bleeding. The rest 198 patients underwent successful laparoscopic cholecystectomy. The surgery was done as per conventional 4-port technique. As per the "American" technique, the surgeon stood to the left of the patient, the first assistant stood to the patient's right and the laparoscopic video camera operator stood to the left of the surgeon. Pneumoperitoneum was established with $\mathrm{CO} 2$ gas using the closed technique through a Veress needle, which was subsequently replaced with a laparoscopic port, placed blindly into the abdominal cavity.

The hepatocystic triangle was dissected, which is the ventral aspect of the area bounded by the gallbladder wall and cystic duct, the liver edge and the common hepatic duct; the cystic artery (And hence Calot's triangle) lies within this space. The hepatocystic triangle was maximally opened and converted into a trapezoid shape by retracting the infundibulum of the gallbladder inferiorly and laterally while maintaining the fundus under traction in a superior and medial direction. After the dissection of the Calot's triangle, the cystic duct was clipped using Ligaclip 300. In 42 patients, the cystic artery was ligated using Ligaclip 300. Three clips were applied, two distally and one proximally on the cystic artery. The cystic artery was divided between the proximal and distal clip. In 156 patients, the artery was coagulated using monopolar cautery with hook. The artery was cauterized just lateral to the lymph node of Calot's. Cautery machine, Schiller Surgitom GD 400 was used. Voltage was 30 watts using spray mode. The divided stumps of cystic duct and artery were ensured for any bile leaks and haemorrhage. The cholecystectomy was completed as per standard procedure, separating the gallbladder out of liver bed with hook monopolar electrocautery and was removed through the epigastric port. Haemostasis was ensured. Suction was done to clear off the debris and blood clots. The cystic duct and artery stump were re-inspected for any bile leak and haemorrhage. Each port incision was infiltrated with bupivacaine for postoperative analgesia. The fascia of the umbilical incision was closed with one large absorbable suture. The other skin incisions were closed using Nylon 2-0.

The patients were observed for any incidences of postoperative haemorrhage and bile leak. All the patients were discharged from the hospital once they were fit to be done so. The hospital stay and any occurrences of post-operative complications were observed and recorded. Patients who developed post-operative complications in our study, mainly port site infections were managed conservatively by adding antibiotics according to culture sensitivity. They were discharged once adequate control of the infection was ensured. All patients were followed up on the 7 th post-operative day.

Among the 201 patients planned for laparoscopic cholecystectomy, 3 had to be converted to open procedure due to development of intraoperative bleeding. All were converted to open cholecystectomy by giving a right subcostal incision and abdomen opened in layers. One patient had uncontrolled bleeding from cystic artery that was getting difficult to control with either surgical clips or electrocoagulation. One patient had bleeding from supraduodenal vein during the procedure. The difficulty in controlling it resulted in turning the case into an open cholecystectomy. The third one had uncontrolled bleeding from liver bed. The liver appeared to be cirrhosed and cauterization was not getting sufficient to control the bleeding, hence had to convert into open procedure. These cases after being opened the complications were adequately dealt with, haemostasis achieved and further closure of laparotomy was done. They were managed and observed post-operatively for complications and discharged on the 5th day as the patients were found fit to be done so and followed up after 1 week.

The results obtained were statistically analysed using SPSS 20.0. Numerical data were expressed as mean+/standard deviation (SD). Observations were done in both the groups to look for any post-operative bleeding, drain bag collection, vital data and ultrasound scan findings were taken into consideration. $\mathrm{P}$ value less than 0.05 was considered statistically significant.

\section{RESULTS AND OBSERVATIONS}

The study contained 201 patients who were planned for laparoscopic cholecystectomy, among which 3 were converted to open cholecystectomy due to intraoperative bleeding. The mean age of the study group was 40.26 years. The M:F sex ratio was 1:4 with 161 females and 40 males. A total of 169 patients, about $85.4 \%$ presented with a diagnosis of chronic cholecystitis. Another 25 patients, about $12.6 \%$ showed a diagnosis of acute on chronic cholecystitis. The rest 4 cases, i.e. $2 \%$ were diagnosed to have acalculous cholecystitis. Among 
the 198 patients who underwent successful laparoscopic cholecystectomy, in 156 patients the artery was coagulated using monopolar cautery with hook, while in 42 patients the cystic artery was ligated using Ligaclip 300. About $86 \%$ (135) patients in electrocautery group and $88 \%$ (37) patients in Ligaclip group were discharged on the first post-operative day itself. No statistical significance was established between the differences. A total of 3 patients (1.5\%) developed postoperative complications, mainly infection at the port site at the $5^{\text {th }}$ postoperative day; 2 of these patients underwent electrocautery and 1 got Ligaclip control of cystic artery. The difference was not statistically significant. Those patients were managed conservatively. There were no reporting of postoperative haemorrhage or bile leak in any of these cases.

\section{DISCUSSION}

Laparoscopic cholecystectomy has been extensively accepted since Mouret first successfully introduced the procedure in 1987. The first laparoscopic cholecystectomy was performed by Muhe in 1986.[11] Now, laparoscopic cholecystectomy is considered to be the gold standard treatment for cholelithiasis.[12] Initially, this new technique was associated with a significant increase in morbidity, in particular iatrogenic biliary injuries and arterial haemorrhage. Anatomical relations on laparoscopic visualization are seen differently as compared to open surgery. So the surgeon has to rely on his sound knowledge of the variations in Calot's triangle and do meticulous dissection in order to avoid injury to the extra-hepatic biliary tree.[7,8] The course and length of the cystic artery in the Calot's triangle is variable.[9,10]

The mean age in study population was 40.26 years. This is in accordance with the studies based on western population.[13]

The M:F ratio in the study was 1:4 constituting 161 females and 40 males. International data suggests that gallstone disease is 3 to 4 times more common in females than males. ${ }^{[14]}$ Female sex, body mass index, low serum high density lipoprotein (HDL) cholesterol levels and positive family history in a first-degree family member represent significant risk factors.[15]

In our study majority of the patients, i.e. $85.4 \%$ amounting about 169 patients presented with a diagnosis of chronic cholecystitis. A total of 25 patients (12.6\%) showed a diagnosis of acute on chronic cholecystitis. The remaining 4 cases, i.e. $2 \%$ were diagnosed to have acalculous cholecystitis. Salman Yousuf Guarya noticed similar results, i.e. 476 (86.7\%) cases presented with chronic cholecystitis, 63 (11.4\%) acute cholecystitis, $6(1 \%)$ mucocele of the gallbladder and $2(0.4 \%)$ had empyema gallbladder.[16] Frequently, chronic cholecystitis presents a large range of associated lesions such as cholesterolosis, muscle hypertrophy, parietal fibrosis, polypoid and adenomatous proliferation of mucous glands, metaplasia, hyperplasia and dysplasia.[17]

During this procedure, the cystic artery can be controlled using surgical clips, harmonic scalpel and ligature or monopolar cautery. The extensive use of surgical clips in laparoscopic surgery has often led to a variety of complications like clip slippage, dislodgement, ulceration, migration, internalization and necrosis of cystic duct with the risk of bile leakage.[18] Harmonic scalpel is also not well used due to its cost effects. Here comes the edge of monopolar electrocautery, it being cheap and easily available. In this study, we tried to compare the outcomes following electrocautery ligation of cystic artery to that with surgical clip (Ligaclip) application.

About $86 \%$ (135) and 88\% (37) patients respectively in electrocautery and Ligaclip group were discharged on the first post-operative day itself. Only $3(1.5 \%)$ patients, 2 in electrocautery and 1 in Ligaclip group developed postoperative port site infection. They were managed conservatively. However, these differences were not statistically significant. Thus, the length of hospital stay and post-operative complications in either group were similar. There were no reports of post-operative haemorrhage or bile leak in either group.

Our findings were in accordance with a study conducted by Katri et al.[19] where they had 158 patients in which they studied the use of monopolar cautery for controlling the cystic artery in 114 patients and clips in 33 patients. They recorded no bleeding complication during the period of the study. They concluded that monopolar cautery can be used to control the cystic artery in laparoscopic cholecystectomy.

Huscher and Colleagues compared the ultrasonic coagulation division of the cystic artery and duct with ligature and found no difference in both methods regarding postoperative mortality and complications. [20]

Redwan AA.[21] conducted a study that included 160 patients undergoing laparoscopic cholecystectomy and compared harmonic scalpel with clips/cautery. They concluded that harmonic scalpel is as effective as the clip/cautery technique in laparoscopic cholecystectomy achieving haemobiliary stasis with shorter operative time.

Soper NJ et al.[22] conducted a study in pigs and assessed the efficacy of monopolar cautery for controlling the cystic duct and artery during laparoscopic cholecystectomy in the pigs. They concluded that monopolar cautery is an efficient method of controlling the artery and duct and no adverse changes in the gall bladder bed or hepatocyte regeneration or cholestasis are observed as evidenced by the pathologic studies carried out in the pigs.

In our study the outcome, mainly hospital stay and postoperative complications obtained were comparable in both groups. The use of monopolar electrocautery instead of surgical clips did not pose any additional risk of post-operative haemorrhage or bile leak. The added advantage with electrocautery was that it did not have the risk of postoperative complications like clip slippage, dislodgement, ulceration, migration, internalization and necrosis of cystic duct with the risk of bile leakage. In other words, it could be stated that monopolar electrocautery is a safer alternative to surgical clips in expert hands. The cost factor and availability makes electrocautery a more feasible option than surgical clips, especially in a low resource setting.

However, with monopolar electrocautery, the depth of burn is less predictable and current can be conducted through non-insulated instruments and trocars. So the laparoscopic surgeon must pay great attention to the anatomical dissection of the Calot's triangle. Excessive and unnecessary dissection or use of electrocautery near the common bile duct should be avoided.[23]

Injury due to electrocautery can be avoided by taking simple precautions like avoiding diathermy near metal clips on the cystic duct and staying close to the gall bladder wall during dissection and control of the cystic artery, preferably lateral to 
the cystic lymph node (Ramirez-Flores et al.).[24] It is important that always short bursts of minimal amounts of energy required to secure homeostasis should be used. In our study we found that the careful use of electrocautery to control the cystic artery has the advantage of shorter operative time.

\section{CONCLUSION}

We conclude that monopolar electrocautery can be used as a safer alternative to surgical clips in control of cystic artery, especially in developing countries where Harmonic scalpel arouse issues of cost factor and affordability. Further randomized control trials will be required to substantiate our findings.

\section{REFERENCES}

1. Soper NJ, Stockmann PT, Dunnegan DL, et al. Laparoscopic cholecystectomy: the new 'gold standard.' Arch Surg 1992;127(8):917-21.

2. Soper NJ, Brunt LM, Kerbl K. Laparoscopic general surgery. N Engl J Med 1994;330:409-19.

3. Conference NC. Gallstones and laparoscopic cholecystectomy. JAMA 1992;269(8):1018-24.

4. Sugerman HJ, Brwer WH, Shiffman M, et al. A multicenter, placebo-controlled, randomized double-blind, prospective trail of prophylactic ursodiol for the prevention of gallstone formation following gastricbypass-induced rapid weight loss. Am J Surg 1995;169(1):91-6.

5. Escarce JJ, Chen W, Schwartz JS. Falling cholecystectomy thresholds since the introduction of laparoscopic cholecystectomy. JAMA 1995;273(20):1581-5.

6. Legorreta AP, Silber JH, Constantino GN, et al. Increased cholecystectomy rate after introduction of laparoscopic cholecystectomy. JAMA 1993;270(12):1429-32.

7. Larobina M, Nottle PD. Extrahepatic biliary anatomy at laparoscopic cholecystectomy: is aberrant anatomy important? ANZ J Surg 2005;75(6):392-5.

8. Nagral S. Anatomy relevant to cholecystectomy. J Mix Access Surg 2005;1(2):53-8.

9. Ayyaz M, Fatima T, Ahmed G. Arterial anatomy in calot's triangle as viewed through the laparoscope. Ann KE Med Coll 2001;7:183-5.

10. Wasserberg N, Gal E, Fuko Z. Surgical clip found in duodenal ulcer after laparoscopic cholecystectomy. Surgical laparoscopy, endoscopy and percutaneous techniques 2003;13(6):387-8.
11. Muhe E. Long-term follow-up after laparoscopic cholecystectomy. Endoscopy 1992;24(9):754-8.

12. Jain SK, Tanwar R, Kaza RC, et al. A prospective, randomized study of comparisonof clipless cholecystectomy with conventional laparoscopic cholecystectomy. J Laparoendosc Adv Surg Tech A 2011;21(3):203-8.

13. Schafmayer C, Hartleb J. Predictors of gall stones composition in 1025 symptomatic gall stones from northern Germany. BMC Gastroenterol 2006;6:36.

14. Heaton KW, Braddon FE, Mountford RA, et al. Symptomatic and silent stones in the community. Gut 1991;32(3):316-20.

15. Nakeeb A, Comuzzie AG, Martin L, et al. Gallstones: genetics versus environment. Ann Surg 2002;235(6):842-9.

16. Guraya SY, Khairy GEA, Murshid KR. Audit of laparoscopic cholecystectomy: 5 years experience in a university hospital. Ann King Edward Med Coll 2004;10:9-10.

17. Stancu M, Caruntu ID, Giusca S, et al. Hyperplasia, metaplasia, dysplasia and neoplasia lesions in chronic cholecystitis-a morphologic study. Rom J Morphol Embryol 2007;48(4):335-42.

18. Zucker KA. Surgical laparoscopy. Philadelphia. Lippincott Williams and Wilkins 2001;2nd Ed:130-41.

19. Katri KM, Bessa SS, Elnagah GA, et al. Is monopolar electrocautery safe and effective for control of the cystic artery during laparoscopic cholecystectomy. J Laparoendosc Adv Surg Tech A 2012;22(6):557-60.

20. Huscher CG, Lirici MM, Di Paola M, et al. Laparoscopic cholecystectomy by ultrasonic dissection without cystic duct and artery ligature. Surg Endosc 2003;17(3):442-51.

21. Redwan AA. Single-working-instrument, double-trocar, clipless cholecystectomy using harmonic scalpel: a feasible, safe and less invasive technique. J Laparoendosc Adv Surg Tech A 2010;20(7):597-603.

22. Soper NJ, Barteau JA, Claymann RV, et al. Safety and efficacy of laparoscopic cholecystectomy using monopolar electrocautery in the porcine model. Surg Laparosc Endosc 1991;1(1):17-22.

23. Strasberg SM. Avoidance of biliary injury during laparoscopic cholecystectomy. J Hepatobiliary Pancreat Surg. 2002;9(5):543-7.

24. Ramirez-Flores R. A new anatomic guide for safe ligation of the cystic artery. Surg Gynecol Obstet 1955;100(5):633-5. 